\title{
Electronic and magnetic properties of Fe clusters inside finite zigzag single-wall carbon nanotubes
}

\author{
F. I. Horga and A. Mañanes* \\ Departamento de Física Moderna, Universidad de Cantabria, E-39005 Santander, Spain
}

\author{
M. J. López and J. A. Alonso \\ Departamento de Física Teórica, Atómica y Óptica, Universidad de Valladolid, E-47011 Valladolid, Spain \\ (Received 15 August 2011; revised manuscript received 7 December 2012; published 4 February 2013)
}

\begin{abstract}
Density functional calculations of the electronic structure of the $\mathrm{Fe}_{12}$ cluster encapsulated inside finite singlewall zigzag carbon nanotubes of indices $(11,0)$ and $(10,0)$ have been performed. Several $\mathrm{Fe}_{12}$ isomers have been considered, including elongated shape isomers aimed to fit well inside the nanotubes, and the icosahedral minimum energy structure. We analyze the structural and magnetic properties of the combined systems, and how those properties change compared to the isolated systems. A strong ferromagnetic coupling between the $\mathrm{Fe}$ atoms occurs both for the free and the encapsulated $\mathrm{Fe}_{12}$ clusters, but there is a small reduction $\left(3-7.4 \mu_{B}\right)$ of the spin magnetic moment of the encapsulated clusters with respect to that of the free ones $\left(\mu=38 \mu_{B}\right)$. The reduction of the magnetic moment is mostly due to the internal redistribution of the spin charges in the iron cluster. In contrast, the spin magnetic moment of the carbon nanotubes, which is zero for the empty tubes, becomes nonzero $\left(1-3 \mu_{B}\right)$ because of the interaction with the encapsulated cluster. We have also studied the encapsulation of atomic $\mathrm{Fe}$ and the growth of small $\mathrm{Fe}_{n}$ clusters $(n=2,4,8)$ encapsulated in a short $(10,0)$ tube. The results suggest that the growth of nanowires formed by distorted tetrahedral $\mathrm{Fe}_{4}$ units will be favorable in $(10,0)$ nanotubes and nanotubes of similar diameter.
\end{abstract}

DOI: 10.1103/PhysRevB.87.085402

PACS number(s): 73.22.-f, 73.63.Fg, 75.75.-c, 85.75.-d

\section{INTRODUCTION}

Carbon nanotubes encapsulating atoms, molecules, or clusters have been synthesized, and their properties have been analyzed, due to their fundamental and technological interest. Nanotubes filled with Fe have potential uses in medicine, ${ }^{1}$ in spintronic devices,${ }^{2}$ including spin valves,${ }^{3}$ and can also be used as probes for magnetic force microscopy. ${ }^{4,5}$ Recent reviews on the preparation of filled carbon nanotubes ${ }^{6,7}$ indicate the activity in the field. In general, free metallic magnetic nanoparticles are oxide coated, but the encapsulation of Fe clusters and nanowires in carbon nanotubes, where these metallic nanostructures are protected from the oxidating environment, ${ }^{8}$ has been shown to be feasible. ${ }^{9,10}$

The aim of this work is to present a theoretical study of the electronic and magnetic properties of iron clusters encapsulated in carbon nanotubes, using density functional theory (DFT). ${ }^{11}$ We shall focus on the $\mathrm{Fe}_{12}$ cluster encapsulated inside finite pieces of single-wall zigzag nanotubes (ZNTs) of indices $(11,0)$ and $(10,0)$. Finite zigzag nanotubes have a peculiar magnetic structure: the ground state is an antiferromagnetic (AFM) spin singlet $S=0$. The local spin polarization oscillates (spin up and spin down) along the direction of the nanotube axis, and it is small, except at the two ends of the nanotube, due to the existence of electronic states localized at the edges of the nanotube. The two ends show opposite orientation of the atomic magnetic moments. ${ }^{12-15}$ Other states are close in energy to the ground state: magnetic states $(M)$ with net total magnetic moment $\mu_{T}$ different from zero (in some of these, the magnetic moments at the two edges show the same orientation ${ }^{13-15}$ ), and a nonmagnetic (NM) state showing local spin compensation along the nanotube. The energy difference $E\left(\mu_{T}^{0}\right)-E(\mathrm{AFM})$ between the lowest-lying magnetic state (with total magnetic moment $\mu_{T}^{0}$ ) and the ground state (AFM) decreases monotonically when the length of the nanotube increases, up to a point where the difference becomes lower than room temperature. ${ }^{14}$ For the shortest zigzag carbon nanotubes, $E\left(\mu_{T}^{0}\right)-E(\mathrm{AFM})$ increases when the diameter of the nanotube increases, showing an oscillating effect. ${ }^{16}$ Our aim is to analyze the interplay between the singular magnetic properties of the finite ZNTs and the large magnetization of the encapsulated Fe clusters. As we have shown in a previous work, ${ }^{15}$ the ZNTs present electronic properties associated to edge states similar to those of zigzag graphene ribbons, ${ }^{17}$ therefore our study could be of relevance in analyzing the properties of proposed magnetoresistive devices based on zigzag graphene ribbons. ${ }^{18}$ Furthermore, as far as finite ZNTs can be considered as organic molecules containing $\pi$ electrons, our results can be useful in the interpretation of the local spin polarization at the organicferromagnetic interface, which has been recently described both theoretically and experimentally. ${ }^{19}$

Previous calculations of the electronic structure of $3 d$ transition-metal atoms and clusters bonded to carbon nanostructures have considered infinitely long carbon nanotubes, ${ }^{20-28}$ an infinite graphene sheet, ${ }^{26,29,30}$ capped finite ZNTs, ${ }^{31,32}$ or finite nanotubes with unsaturated open ends. ${ }^{33}$ The effects due to the edge states have not been analyzed in those works. The calculations indicate that the magnetism is confined within the Fe aggregates, with a small reduction of the magnetic moment per atom with respect to the free $\mathrm{Fe}$ clusters. On the other hand, the magnetic moments per atom result enhanced with respect to those of bulk $\mathrm{Fe}$, due to the reduced coordination of the metal atoms. In the particular case of iron aggregates encapsulated in finite $(8,0)$ nanotubes, the calculations ${ }^{33}$ indicate that the cluster is ferromagnetic, with a total magnetic moment which is larger for the capped tubes than for those with unsaturated open ends. Fujima and $\mathrm{Oda}^{32}$ have studied iron chains encapsulated in finite capped $(5,0)$ and $(8,0)$ ZNTs. They found that the magnetism 
of the encapsulated $\mathrm{Fe}$ atoms is not enhanced, in general. Calculations for $\mathrm{Fe}$ nanowires inside infinite nanotubes ${ }^{24}$ predict ferromagnetic ordering in the iron structure when the ratio between the diameters of the nanowire and the nanotube is small, and a tendency towards AFM ordering when the iron wire fits tightly into the nanotube.

The finite nanotubes studied in previous works ${ }^{32,33}$ are different from those considered here. Now, the two ends of the ZNTs are opened and hydrogen atoms saturate the dangling bonds of the carbon atoms at the ends. We have selected the systems $\mathrm{Fe}_{12} @ \mathrm{C}_{88} \mathrm{H}_{22}$ and $\mathrm{Fe}_{12} @ \mathrm{C}_{132} \mathrm{H}_{22}$ for the $(11,0)$ and $\mathrm{Fe}_{12} @ \mathrm{C}_{80} \mathrm{H}_{20}$ and $\mathrm{Fe}_{12} @ \mathrm{C}_{120} \mathrm{H}_{20}$ for the $(10,0)$ ZNT to discuss both the radius and length dependence of the properties of the combined systems and because they have an iron/carbon ratio which is analogous to those considered in the quoted previous calculations. After presenting in Sec. II the method used in the calculations, based on DFT, we next show our results and their analysis. We describe the properties of the $\mathrm{Fe}_{12}$ cluster and other smaller $\mathrm{Fe}_{n}$ clusters $(n=2$, 4 , and 8), and of the H-terminated finite $(11,0)$ and $(10,0)$ nanotubes in Sec. III, and then we turn in Sec. IV to $\mathrm{Fe}_{n}$ encapsulated in these nanotubes, with emphasis on $\mathrm{Fe}_{12}$. We pay special attention to the magnetic properties. In the last part of Sec. IV we discuss the evolution of the stability and the magnetic properties with the size of the aggregate, calculating the equilibrium structures of $\mathrm{Fe}_{n} @ \mathrm{C}_{80} \mathrm{H}_{20}$ for $n=1,2$, 4, and 8 . This study gives information on the growth of the Fe cluster inside the nanotubes. The conclusions are given in Sec. V. A relevant result is that there is only a small reduction of the total magnetic moments of the $\mathrm{Fe}$ aggregates encapsulated in ZNTs, with respect to those of the free clusters. However, the reduction of the magnetic moment of the iron cluster is combined with a net magnetization of the carbon structure.

\section{METHOD}

We perform DFT calculations ${ }^{11}$ using the ADF 2009.01 code $^{34,35}$ with the revPBE generalized gradient approximation $^{36}$ (GGA) for electronic exchange and correlation. Basis sets formed by Slater-type atomic orbitals are employed to construct the molecular orbitals. All the electrons are included in the calculations, but a frozen core approximation is used for the internal electrons of each atom: a frozen heliumlike core for $\mathrm{C}$ and an argonlike core for $\mathrm{Fe}$. For the valence electrons of $\mathrm{C}$ and $\mathrm{H}$, we have used a triple- $\zeta$ basis with one polarization function (TZP). It contains four $s$ orbitals, three sets of $p$ and one set of $d$ orbitals for each carbon, and three $s$ orbitals and one set of $p$ orbitals for each hydrogen. For the iron atoms, we use a TZ2P basis composed by six $s$ orbitals, three sets of $p$ orbitals, four sets of $d$ orbitals, and one set of $f$ orbitals. The code incorporates the molecular symmetry of the system using orthogonalized symmetryadapted molecular orbitals to solve the Kohn-Sham equations. The atomic positions are optimized using a conjugate gradient method where the energy and the atomic forces are minimized. In general, we ask for an accuracy of at least $10^{-4}$ hartree for the energies and $10^{-3}$ hartree $/ \AA$ for the energy gradients. For spin-unrestricted calculations, as in the present case, it is possible in the code to tune the initial potential by allowing for different spin configurations even for equivalent atoms, thus the code can perform spin broken-symmetry calculations. The magnetic structure will be analyzed using the Mulliken charges per atom $n_{\alpha}$ and $n_{\beta}$ for majority $(\alpha)$ and minority $(\beta)$ spin electrons. The atomic magnetic moments are calculated as $\mu=\left(n_{\alpha}-n_{\beta}\right) \mu_{B}$, in units of the Bohr magneton $\mu_{B}$, and the total magnetic moments are given by $\mu_{T}=\left(N_{\alpha}-N_{\beta}\right) \mu_{B}$, where $N_{\alpha}=\sum n_{\alpha}$ and $N_{\beta}=\sum n_{\beta}$ are the total numbers of majority and minority spin electrons, respectively. The total spin is obtained as $S=\left(N_{\alpha}-N_{\beta}\right) / 2$.

\section{IRON CLUSTERS AND FINITE NANOTUBES AS ISOLATED AGGREGATES}

\section{A. Fe clusters}

Transition-metal clusters in matrices, or deposited on surfaces, have a magnetic moment significantly enhanced relative to the bulk. On the other hand, the magnetic moments are smaller than in the free clusters. ${ }^{37}$ For small free $\mathrm{Ni}$ and Fe clusters, spin plays a role in determining the most stable structures, and compact structures are more stable than open ones. ${ }^{38,39}$ In the case of Fe-filled narrow carbon nanotubes, the structure of the cluster has to adapt to the diameter of the nanotube, and elongated iron clusters are expected. This is confirmed by experiments using high-resolution electron microscopy, ${ }^{40}$ showing that there is not free space between the metal and the carbon walls and that elongated iron aggregates condensate separated by empty regions along the nanotube. ${ }^{6,40}$ For this reason, we shall focus on an isomer of $\mathrm{Fe}_{12}$ having an elongated geometry that fits well inside the $(10,0)$ and $(11,0)$ ZNTs considered in this paper. Moreover, we will investigate the icosahedral structure of $\mathrm{Fe}_{12}$ and a second elongated isomer based on tetrahedral units.

To obtain an elongated structure for $\mathrm{Fe}_{12}$, we have started with an optimized linear chain. The analysis of the vibrational properties showed that the linear $\mathrm{Fe}_{12}$ chain is a saddle point, and not a local minimum. Displacing the atoms following one of the imaginary frequency modes we have obtained, after a complete relaxation, the three-dimensional (3D) structure given in Fig. 1. A trend towards fivefold coordination is observed, and the geometry can be described as two pentagonal bipyramids with a common edge (between atoms Fe1 and $\mathrm{Fe} 2$ in Fig. 1), and bent with respect to that edge, so that

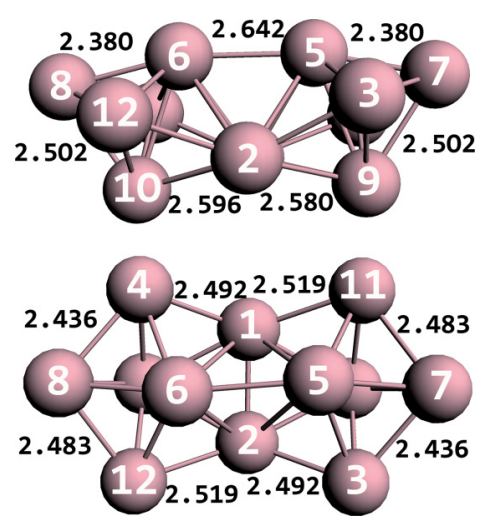

FIG. 1. (Color online) Two lateral views of the geometrical structure of the elongated isomer of $\mathrm{Fe}_{12}$ with total magnetic moment $\mu_{T}=38 \mu_{B}$. The interatomic distances are given in $\AA$. 
TABLE I. Structural and electronic properties of isolated $\mathrm{Fe}_{n}$ aggregates. $d_{m}$ and $d_{M}$ are the minimum and maximum distances between neighbor atoms, $\overline{E_{b}^{0}}=E_{b}^{0} / n$ is the binding energy per atom, $\Delta \epsilon$ is the HOMO-LUMO gap for each spin orientation, $\mu_{T}$ is the total magnetic moment, and $\overline{\mu_{T}}$ is the magnetic moment per atom.

\begin{tabular}{lcccccc}
\hline \hline $\begin{array}{l}\mathrm{Fe}_{n} \\
n\end{array}$ & $\begin{array}{c}d_{m} \\
(\AA)\end{array}$ & $\begin{array}{c}d_{M} \\
(\AA)\end{array}$ & $\begin{array}{c}\overline{E_{b}^{0}} \\
(\mathrm{eV})\end{array}$ & $\begin{array}{c}\Delta \epsilon_{\alpha} \\
(\mathrm{eV})\end{array}$ & $\begin{array}{c}\Delta \epsilon_{\beta} \\
(\mathrm{eV})\end{array}$ & $\begin{array}{c}\mu_{T} \\
\mu_{T} \\
\left(\mu_{B}\right)\end{array}$ \\
\hline 12(icosahedral) & 2.38 & 2.92 & 3.173 & 0.20 & 0.43 & 38 \\
12(tetrahedral chain) & 2.36 & 2.89 & 3.104 & 0.66 & 0.43 & 3.17 \\
12(elongated) & 2.37 & 2.74 & 3.085 & 0.82 & 0.09 & 38 \\
8(two tetrahedra) & 2.32 & 2.76 & 2.996 & 1.57 & 0.49 & 24 \\
4(linear) & 2.11 & 2.69 & 1.789 & 2.17 & 0.07 & 14 \\
4(tetrahedron) & 2.30 & 2.63 & 2.306 & 0.51 & 0.57 & 3.17 \\
2 & 2.02 & 2.02 & 1.612 & 1.11 & 0.71 & 3.5 \\
\hline \hline
\end{tabular}

the apical atoms Fe5 and Fe6 are closer to each other than the other two apical atoms Fe9 and Fe10. This structure is a true local minimum. Some relevant structural and electronic properties of the cluster, the interatomic distances, binding energy, electronic energy gaps, and total magnetic moment are given in Table I. The binding energy

$$
E_{b}^{0}\left(\mathrm{Fe}_{n}\right)=n E(\mathrm{Fe})-E\left(\mathrm{Fe}_{n}\right),
$$

where $E(\mathrm{Fe})$ and $E\left(\mathrm{Fe}_{n}\right)$ represent the total energies of the free atom and the cluster, respectively ( $n=12$ in this case), is $3.085 \mathrm{eV} /$ atom. The nearest-neighbor interatomic distances $d_{n n}$ vary between 2.37 and $2.74 \AA$. The gaps are obtained as the difference between the energies of the lowest unoccupied molecular orbital (LUMO) and the highest occupied molecular orbital (HOMO) for each spin orientation. The total magnetic moment is $\mu_{T}=38 \mu_{B}$, or $3.17 \mu_{B}$ /atom. For comparison, the interatomic distance, the cohesive energy, and the magnetic moment of bulk iron are $d_{n n}($ bulk $)=2.48 \AA, E_{b}($ bulk $)=$ $4.28 \mathrm{eV} /$ atom, and $\mu$ (bulk) $=2.2 \mu_{B}$ atom. $^{41}$

We will consider this $\mathrm{Fe}_{12}$ isomer as representative of the elongated structures that appear in iron-filled carbon nanotubes (CNTs). Experimentally, ${ }^{6,7}$ the filling can be performed using in situ or ex situ methods. In the first case, the iron nanostructure inside the tube forms simultaneously with the process of nanotube growth, like in chemical vapor deposition in the presence of an Fe precursor. In the ex situ methods, nanotubes are filled by capillarity from a suspension of nanoparticles. ${ }^{7}$ The aim of this work is to investigate the properties of ironfilled nanotubes rather than the growth mechanisms. However, we present in Sec. IV C a brief investigation of the first steps of the filling of tubes with iron atoms and small clusters. With this purpose in mind, we report in Table I the calculated properties of the free $\mathrm{Fe}_{2}, \mathrm{Fe}_{4}$, and $\mathrm{Fe}_{8}$ clusters, and of an $\mathrm{Fe}_{12}$ isomer based on tetrahedral units. In agreement with calculations of other authors, ${ }^{39}$ we find that the minimum energy structure of $\mathrm{Fe}_{12}$ is a, slightly distorted, centered icosahedron with a missing vertex. Its binding energy is $3.173 \mathrm{eV} /$ atom. It is interesting to notice that, despite the structural differences, the total magnetic moment $\mu_{T}=38 \mu_{B}$ is the same as those of the elongated isomer and the isomer based on tetrahedral units.

The electronic density of states (DOS) of the elongated $\mathrm{Fe}_{12}$ isomer is shown in Fig. 2, separating the $s, p$, and $d$ components. The most relevant feature is the existence of a gap at the Fermi level for the majority $\alpha$-spin electrons, and a very small gap for the minority $\beta$-spin electrons. The HOMO and LUMO states for the majority spin have $s p$ character. For minority spin, both frontier orbitals have $d$ character. The spatial distributions of the HOMO and LUMO orbitals are shown in the insets of the figure. The $d$ band for the majority spin electrons lies in the region between -8.5 and $-4 \mathrm{eV}$, and it is fully occupied. For minority spin, the $d$ band is upward shifted by $2 \mathrm{eV}$ with respect to the $\alpha$-spin $d$ band, it is partially occupied, and it shows a substantial density of states under the Fermi level.

\section{B. Finite zigzag carbon nanotubes}

We have considered two finite pieces of the $(11,0)$ and the $(10,0)$ zigzag single-wall carbon nanotubes. The short and long pieces of the $(m, 0)$ tubes are formed, respectively, by four and six zigzag rings of $2 m$ carbon atoms each and $m$ hydrogen atoms saturating the carbon atoms on each end side of the tube. The tube lengths, from carbon to carbon, are 7.12 and $11.40 \AA$, for the short and long tubes, respectively. The

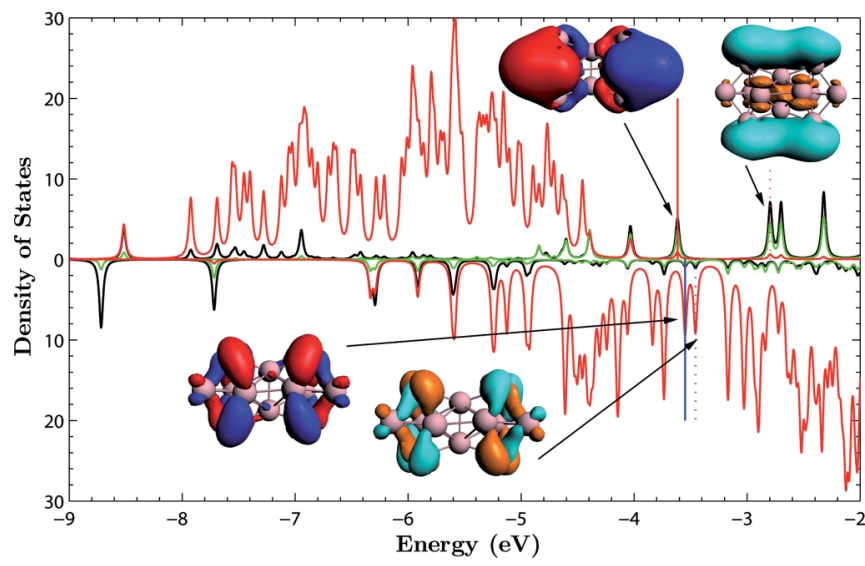

FIG. 2. (Color online) Electronic density of states, in arbitrary units, for the elongated isomer of $\mathrm{Fe}_{12}$ given in Fig. 1. Upper curves correspond to majority spin, and lower curves to minority spin. Red curves indicate $d$ states, green curves $p$ states, and black curves $s$ states. The states have been broadened with Lorenztians of width $\Gamma=0.04 \mathrm{eV}$. Vertical continuous and dashed lines indicate positions of the HOMO and LUMO, respectively. The insets give the HOMO and LUMO wave functions. 


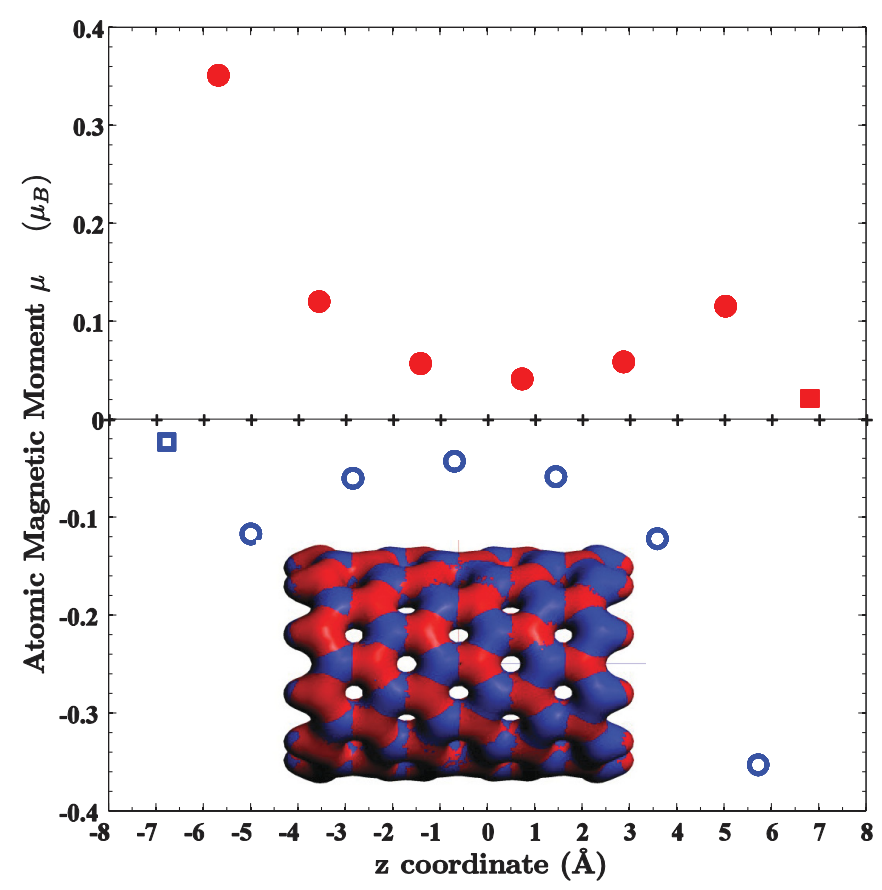

FIG. 3. (Color online) Atomic magnetic moments for the long $(10,0) \mathrm{ZNT}$ as a function of the coordinate $z$ along the nanotube axis. Due to the symmetry of the system, atoms with the same $z$ coordinate have the same value of the magnetic moment. Filled and empty circles correspond to $\mathrm{C}$ atoms, and squares to $\mathrm{H}$ atoms. The leftmost $\mathrm{C}$ atoms are dominated by $\alpha$ spin and the rightmost $\mathrm{C}$ atoms by $\beta$ spin. The inset gives the isosurfaces for the spin densities where $\rho_{\alpha}(\vec{r})=0.025$ a.u. (red) and $\rho_{\beta}(\vec{r})=0.025$ a.u. (blue). The surface shown corresponds to the most external one, then a red (blue) color indicates that the atoms have excess $\alpha(\beta)$ population.

diameters are $8.62 \AA$ for $(11,0)$, and $7.83 \AA$ for $(10,0)$. These finite ZNTs can be considered as aromatic molecules $\mathrm{C}_{88} \mathrm{H}_{22}$ for the short $(11,0), \mathrm{C}_{132} \mathrm{H}_{22}$ for the long $(11,0), \mathrm{C}_{80} \mathrm{H}_{20}$ for the short $(10,0)$, and $\mathrm{C}_{120} \mathrm{H}_{20}$ for the long $(10,0)$ nanotubes.

As for other finite ZNTs, ${ }^{15}$ the ground state presents an AFM spin configuration along the tube, with zero total magnetic moment $\left(\mu_{T}=0\right)$. As an example, the spin structure of the ground state of the long $(10,0)$ ZNT is given in Fig. 3, where the atomic magnetic moments are given for the carbon atoms along the tube axis ( $z$ direction). The AFM coupling means that positive and negative values of the spin polarization alternate along the $z$ direction. The AFM structure is seen in the inset of Fig. 3, where we present the spin densities. The spin polarization is enhanced on the carbon atoms at the ends of the nanotube. The picture is similar for the other ZNTs. The HOMO-LUMO gaps have substantial values of 0.67 and $0.64 \mathrm{eV}$ for the short and long $(11,0)$ ZNTs and of 0.64 and $0.59 \mathrm{eV}$ for the corresponding $(10,0)$ tubes. The $\alpha$ and $\beta$ gaps are identical within each tube. The trend of our results agrees with density functional calculations ${ }^{14}$ which indicate that the electronic gap of finite ZNTs decreases as the length of the nanotube increases.

The electronic density of states of the long $(10,0) \mathrm{ZNT}$, with six zigzag rings, is shown in Fig. 4. In the energy range considered in the figure, all the states are of dominant $p$ character. Most of the states are doubly degenerate

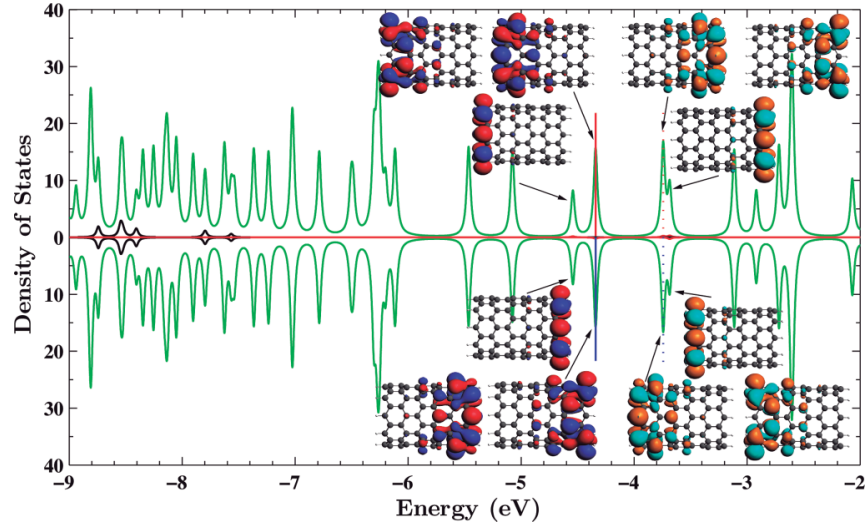

FIG. 4. (Color online) Electronic density of states, in arbitrary units, for the finite $(10,0)$ ZNT with six zigzag rings (long ZNT) in the AFM ground state with $\mu_{T}=0$. Upper curves for $\alpha$ spin, lower curves for $\beta$ spin. Green curves indicate $p$-like states, and black curves $s$-like states. All states have been broadened as in Fig. 2. Vertical continuous lines mark the HOMO and vertical dashed lines the LUMO. Insets give the wave functions of the HOMO and LUMO (double degenerate), and of the nondegenarate HOMO-1 and $\mathrm{LUMO}+1$.

( $E$ symmetry), in particular the HOMO and LUMO. The wave functions of these states, shown as insets, are localized at the ends of the nanotube. There are two other localized states: the nondegenerate ( $A$ symmetry) $\mathrm{HOMO}-1$ and LUMO+1, and their wave functions are also given in Fig. 4. They complete the edge states of the finite zigzag nanotube. All the wave functions corresponding to the other occupied and virtual states are delocalized along the ZNT axis. It is clear in Fig. 4 that the DOS presents spin compensation at any energy.

These finite ZNTs have a variety of low-lying magnetic states with total magnetic moment $\mu_{T}>0$, and also a nonmagnetic state $\left(\mu_{T}=0\right)$. For the shortest ZNTs with four zigzag carbon rings, we present in Fig. 5 the energy difference

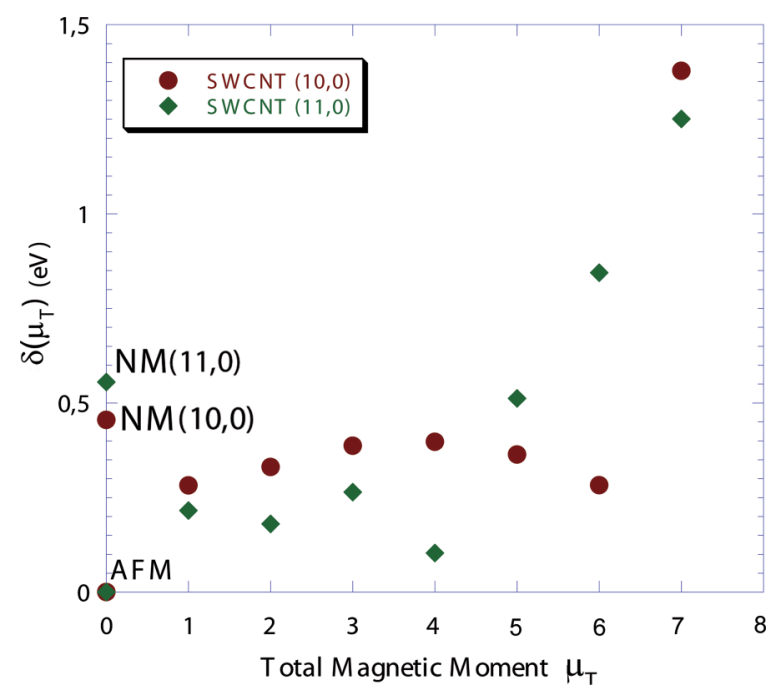

FIG. 5. (Color online) Difference between the energy of lowlying magnetic states with magnetic moment $\mu_{T}$ and the AFM ground state, as a function of $\mu_{T}$, for the short $(11,0)$ and $(10,0)$ nanotubes. The location of the nonmagnetic states, NM, is also indicated. 
between several of those states and the AFM ground state:

$$
\delta\left(\mu_{T}\right)=E\left(\mu_{T}\right)-E(\mathrm{AFM}) .
$$

In the $(10,0)$ nanotube, the lowest energy difference $\delta\left(\mu_{T}^{0}\right)=$ $0.28 \mathrm{eV}$ occurs for two low-lying magnetic states with $\mu_{T}=$ $1 \mu_{B}$ and $6 \mu_{B}$. In the state with $\mu_{T}=1 \mu_{B}$, the local distribution of magnetic moments is analogous to that shown in Fig. 4, that is, an AFM coupling along the tube with the edges having opposite spins. However, there is no total compensation of the spins. On the other hand, the state with $\mu_{T}=6 \mu_{B}$ has a peculiar magnetic structure: the distribution of spins is AFM on each half of the tube (left and right), and starts with the same spin orientation $(\alpha)$ at both ends. Consequently, the AFM coupling becomes frustrated in the middle part of the tube. In the case of the $(11,0)$ nanotube, a state with $\mu_{T}=4 \mu_{B}$ shows the lowest energy difference $\delta\left(\mu_{T}^{0}\right)=0.10 \mathrm{eV}$. This state also has a frustrated AFM distribution of spins. Hod and Scuseria ${ }^{14}$ have shown that $\delta\left(\mu_{T}^{0}\right)$ decreases sharply for long finite ZNTs, becoming lower than the thermal energy at room temperature $(0.025 \mathrm{eV})$ in most cases. One of the aims of this work is to analyze the changes induced in the magnetic state of the nanotubes by the presence of an encapsulated iron cluster; we discuss this topic in the next section.

\section{ENCAPSULATION OF IRON CLUSTERS IN FINITE CARBON NANOTUBES}

\section{A. $\mathrm{Fe}_{12}$ inside finite $(\mathbf{1 1 , 0 )}$ nanotubes}

Figure 6 shows two views of the structure of the short $(11,0)$ tube with the encapsulated cluster. The equilibrium geometry has been obtained starting with the elongated structure of the iron cluster given in Fig. 1, centered inside the nanotube, and allowing for full relaxation of the whole system, without imposing any symmetry restrictions. The iron cluster retains the structure of Fig. 1 with only small distortions: The apical atoms $\mathrm{Fe} 5$ and $\mathrm{Fe} 6$ are slightly closer than in the free aggregate, and the other two apical atoms, Fe9 and Fe10, are more separated. The minimum distance between $\mathrm{Fe}$ and $\mathrm{C}$ atoms is $2.21 \AA$, and the five $\mathrm{Fe}$ atoms closer to the walls (only five $\mathrm{Fe}$ atoms have $\mathrm{C}$ neighbors at distances between 2.21 and $2.70 \AA$ ) prefer top and bridge locations with respect to the $\mathrm{C}$ atoms. The final position of the cluster is shifted from its initial centered location towards one end of the nanotube. The nanotube is a bit flattened by its interaction with the cluster, and the deformation has been estimated by considering the minimum $R_{\min }$ and maximum $R_{\max }$ distances of the
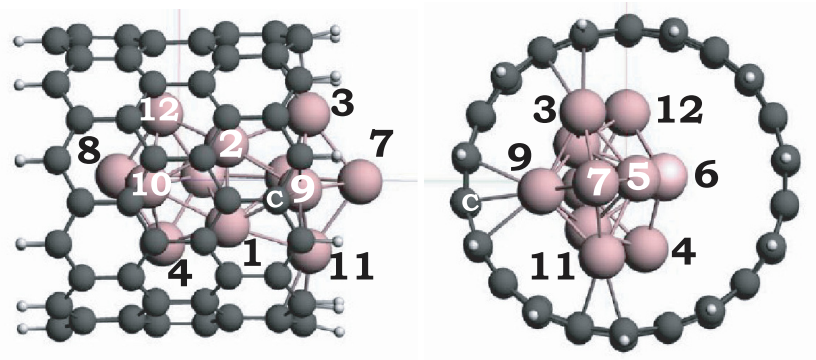

FIG. 6. (Color online) Lateral and front views of the optimized structure of the $\mathrm{Fe}_{12}$ cluster encapsulated in the short $(11,0)$ nanotube. carbon atoms to the nanotube axis, obtaining an eccentricity $e_{c c}=\sqrt{1-\left(R_{\min } / R_{\max }\right)^{2}}=0.27$. The magnetic moment of the system (cluster plus nanotube) is $\mu_{T}=36 \mu_{B}$, a little reduced from the value $\mu_{T}=38 \mu_{B}$ of the free iron cluster. The iron atoms have positive magnetic moments, slightly lower than those in the free aggregate, and the ZNT has a positive magnetic moment of $1 \mu_{B}$.

To characterize the stability of the $\mathrm{Fe}_{n}$ cluster inside the nanotube, we use the binding energy of the encapsulated cluster

$$
E_{b}\left(\mathrm{Fe}_{n} @ \mathrm{C}_{x} \mathrm{H}_{y}\right)=E\left(\mathrm{C}_{x} \mathrm{H}_{y}\right)+n E(\mathrm{Fe})-E\left(\mathrm{Fe}_{n} @ \mathrm{C}_{x} \mathrm{H}_{y}\right),
$$

which is given in Table II. $E_{b}$ is the heat released by starting with the free $\mathrm{Fe}$ atoms and forming the $\mathrm{Fe}_{n}$ cluster inside the nanotube. It can also be seen as the energy necessary to separate the system into a clean nanotube and $n$ isolated free Fe atoms. The binding energy is positive, so it is energetically favorable to introduce the $12 \mathrm{Fe}$ atoms into the finite nanotube. The binding energy in the short $(11,0)$ nanotube is $36.85 \mathrm{eV}$ (3.071 eV per Fe atom), a value slightly smaller than the binding energy of the isolated iron cluster $E_{b}^{0}\left(\mathrm{Fe}_{12}\right)=37.02 \mathrm{eV}$, but the difference is marginal. However, it is energetically very favorable to introduce iron atoms inside the nanotubes, as we shall discuss in Sec. IV C. Those encapsulated atoms have a strong tendency to aggregate and form clusters.

To discuss the influence of the length of the nanotube, we have enlarged the ZNT from four to six zigzag rings. The system was fully relaxed to obtain the equilibrium structure of $\mathrm{Fe}_{12} @ \mathrm{C}_{132} \mathrm{H}_{22}$. The total magnetic moment of the system is $\mu_{T}=36 \mu_{B}$, the same value as in the shorter tube, and the binding energy of the cluster to the nanotube is $E_{b}=$ $35.28 \mathrm{eV}$. The binding energy is $1.57 \mathrm{eV}$ smaller compared to encapsulation in the shorter nanotube because of the lower interaction with the edge of the tube. The binding energy per atom and other indices of stability, such as the HOMO-LUMO gap, are indicated in Table II. For the longer nanotube, we obtain smaller $\alpha$ and $\beta$ gaps.

The upper panel of Fig. 7 shows the total electronic DOS for majority and minority spins in the system formed by $\mathrm{Fe}_{12}$ encapsulated in the short $(11,0) \mathrm{ZNT}$. The DOS for the majority spin shows a gap of $0.32 \mathrm{eV}$. The gap for the minority spin is smaller, $0.135 \mathrm{eV}$. The middle and bottom panels report the projections of the DOS on the two subsystems: iron cluster and carbon nanotube; in each case, the DOS is separated in $s$-, $p$-, and $d$-like contributions. The DOS projected on the $\mathrm{Fe}$ cluster is almost identical to the DOS of the free aggregate, as can be seen by comparison with Fig. 2. In contrast, comparison of the DOS projected on the ZNT, the bottom panel of Fig. 7, with the DOS of the clean nanotube, which is similar to the one presented in Fig. 4 for the long $(10,0)$ tube, indicates a strong modification of the DOS of the nanotube. The electronic states projected on the ZNT have lost the twofold degeneracy that was present in the clean nanotube. The $\alpha-\beta$ spin cancellation is not preserved for the states with energies above $-8.5 \mathrm{eV}$, and this is due to the hybridization between the tube and iron states. The analysis of the DOS shows hybridization between the $\alpha$ states of cluster and tube around the Fermi level, and between a few $d$ - $\alpha$ states of $\mathrm{Fe}$ and $p-\alpha$ states of $\mathrm{C}$ in the 
TABLE II. Binding energy per iron atom $\overline{E_{b}} \equiv E_{b} / n$, electronic HOMO-LUMO gaps, $\Delta \epsilon_{\alpha}$ and $\Delta \epsilon_{\beta}$, total magnetic moment $\mu_{T}$ of the system $\mathrm{Fe}_{n} @(m, 0)$, and change in the total magnetic moment $\Delta \mu_{T}$ with respect to the isolated $\mathrm{Fe}_{n}$ and $(m, 0)$ ZNT. For the elongated $\mathrm{Fe}_{12} @(m, 0)$, the first and second lines correspond to the short and long nanotubes, respectively.

\begin{tabular}{|c|c|c|c|c|c|}
\hline $\mathrm{Fe}_{n} @(\mathrm{~m}, 0)$ & $\begin{array}{c}\overline{E_{b}} \\
(\mathrm{eV})\end{array}$ & $\begin{array}{c}\Delta \epsilon_{\alpha} \\
(\mathrm{eV})\end{array}$ & $\begin{array}{c}\Delta \epsilon_{\beta} \\
(\mathrm{eV})\end{array}$ & $\begin{array}{c}\mu_{T} \\
\left(\mu_{B}\right)\end{array}$ & $\begin{array}{l}\Delta \mu_{T} \\
\left(\mu_{B}\right)\end{array}$ \\
\hline \multirow[t]{2}{*}{$\mathrm{Fe}_{12} @(11,0)$ elongated } & 3.071 & 0.321 & 0.135 & 36 & -2 \\
\hline & 2.940 & 0.076 & 0.122 & 36 & -2 \\
\hline \multirow[t]{2}{*}{$\mathrm{Fe}_{12} @(10,0)$ elongated } & 3.052 & 0.344 & 0.141 & 36 & -2 \\
\hline & 2.885 & 0.535 & 0.035 & 36 & -2 \\
\hline $\mathrm{Fe}_{12} @(10,0)$ icosahedral & 3.172 & 0.203 & 0.083 & 32 & -6 \\
\hline $\mathrm{Fe}_{12} @(10,0)$ tetrahedral chain & 3.057 & 0.186 & 0.211 & 34 & -4 \\
\hline $\mathrm{Fe}_{8} @(10,0)$ two tetrahedra & 2.885 & 0.242 & 0.153 & 26 & +2 \\
\hline $\mathrm{Fe}_{4} @(10,0)$ chain & 1.883 & 0.178 & 0.345 & 14 & 0 \\
\hline $\mathrm{Fe}_{4} @(10,0)$ tetrahedron centered & 2.338 & 0.152 & 0.192 & 14 & 0 \\
\hline $\mathrm{Fe}_{4} @(10,0)$ tetrahedron on edge & 2.616 & 0.161 & 0.375 & 14 & 0 \\
\hline $\mathrm{Fe}_{2} @(10,0)$ & 1.785 & 0.517 & 0.480 & 6 & 0 \\
\hline $\mathrm{Fe} @(10,0)$ top (on edge $\mathrm{C}$ ) & 1.454 & 0.372 & 0.594 & 2 & -2 \\
\hline Fe@(10,0) hollow & 0.947 & 0.541 & 0.498 & 2 & -2 \\
\hline
\end{tabular}

energy range between -5.8 and $-8.5 \mathrm{eV}$. With respect to the $\beta$ band, there is some hybridization at the $\beta$-HOMO, and for a few states in the energy range from -3.8 to $-5.6 \mathrm{eV}$ and around $-6 \mathrm{eV}$. The occupied hybrid orbitals reflect the bonding between the Fe cluster and the nanotube. A similar DOS is found for $\mathrm{Fe}_{12}$ inside the longer $(11,0)$ nanotube, with the noticeable difference of a low $\alpha$ gap, as is given in Table II.

Some charge transfer from the iron cluster to the carbon nanotube can be expected due to the energy matching between the HOMO states of $\mathrm{Fe}_{12}$ and the LUMO states of the pure ZNT. To evaluate this charge transfer, we have calculated the difference between the Mulliken atomic charges of the combined system and those of the isolated systems. This analysis indicates a transfer of 0.43 electrons from the $\mathrm{Fe}_{12}$ aggregate to the short $(11,0) \mathrm{ZNT}$. This trend is corroborated by other population analyses. The Voronoi charges ${ }^{34}$ indicate a transfer of 0.56 electrons from the iron cluster to the nanotube. However, the reduction of $2 \mu_{B}$ in the total magnetic moment $\mu_{T}$ with respect to the isolated systems is not only due to the charge transfer. Table III gives the change in the magnetic moments (with respect to the isolated systems) of the Fe cluster and the ZNT. In the present case, $\mathrm{Fe}_{12} @(11,0)$ (short ZNT), the reduction in the iron cluster is $\Delta \mu=-3.01 \mu_{B}$, and the enhancement in the ZNT is $\Delta \mu=1.01 \mu_{B}$. The changes in the magnetic moments are due, partially, to the charge transfer and, to a larger extent, to the internal redistribution of the $\alpha$ and $\beta$ charges both in the $\mathrm{Fe}$ atoms which are closer to the ZNT walls and in the carbon atoms of the ZNT.

Further insight on the distribution of spins of the composite system can be accessed from the atomic magnetic moments, shown in Fig. 8 as a function of the $z$ coordinate of each atom along the nanotube axis. As expected, the iron atoms show the dominant atomic magnetic moments, although these experience a small reduction from their values in the free cluster. The largest reduction occurs for the Fe atoms closest to the walls of the nanotube. Consequently, the partial magnetic moment corresponding to the encapsulated iron cluster is around $\mu=35 \mu_{B}$, smaller than $\mu=38 \mu_{B}$ of the free $\mathrm{Fe}_{12}$. The changes on the carbon atoms are qualitatively important.
Now the magnetic moment corresponding to the carbon nanotube is $1.01 \mu_{B}$, in contrast with the zero value in the clean nanotubes. The original antiferromagnetic spin ordering of the free nanotube along the axial direction becomes perturbed by the presence of the encapsulated Fe cluster. The change in magnetization of the carbon structure can be seen as the result of a spin injection from the iron cluster to the ZNT. This induced magnetization is similar to that recently obtained, both experimentally and by DFT calculations, in the interaction of aromatic molecules with a ferromagnetic substrate. ${ }^{19}$

\section{B. $\mathrm{Fe}_{12}$ cluster inside $(\mathbf{1 0 , 0})$ nanotubes}

We have also studied the properties of $\mathrm{Fe}_{12}$ inside a narrower nanotube, a finite $(10,0)$ ZNT with diameter $7.83 \AA$. As in the previous case, we have considered first a short nanotube, formed by four zigzag $\mathrm{C}$ rings, and then a longer nanotube, formed by six zigzag $C$ rings. Starting with the elongated $\mathrm{Fe}_{12}$ isomer centered inside the short ZNT, the final relaxed geometry of $\mathrm{Fe}_{12}$ is analogous to that described for the $(11,0)$ tube: the iron cluster is slightly displaced towards one of the ends. In a second simulation, we have used the enlarged carbon nanotube, and the final structure is given in Fig. 9. There are seven $\mathrm{Fe}$ atoms which have $\mathrm{C}$ neighbors at distances between 2.16 and $2.70 \AA$. The total number of $\mathrm{C}$ atoms at those distances is now 27 , much larger than the $18 \mathrm{C}$ neighbors in the same range of distances for $\mathrm{Fe}_{12}$ encapsulated in the long $(11,0)$ ZNT. The iron cluster undergoes a small distortion with respect to the free aggregate, it becomes more elongated, and the two twinned pentagonal bipyramids have their vertical axes better aligned in parallel: atoms $\mathrm{Fe} 9$ and $\mathrm{Fe} 10$ in Fig. 9 are closer than in the free cluster. The nanotubes undergo small ellipsoidal deformations, with eccentricities $e_{c c}=0.36$ and 0.26 for the short and long nanotubes, respectively.

The binding energies of the encapsulated cluster and the $\alpha$ and $\beta$ electronic gaps are given in Table II. The binding energies of $\mathrm{Fe}_{12}$ inside the $(10,0)$ nanotubes are slightly smaller than the corresponding binding energies inside $(11,0)$ nanotubes of the same length. The lower binding energies arise 

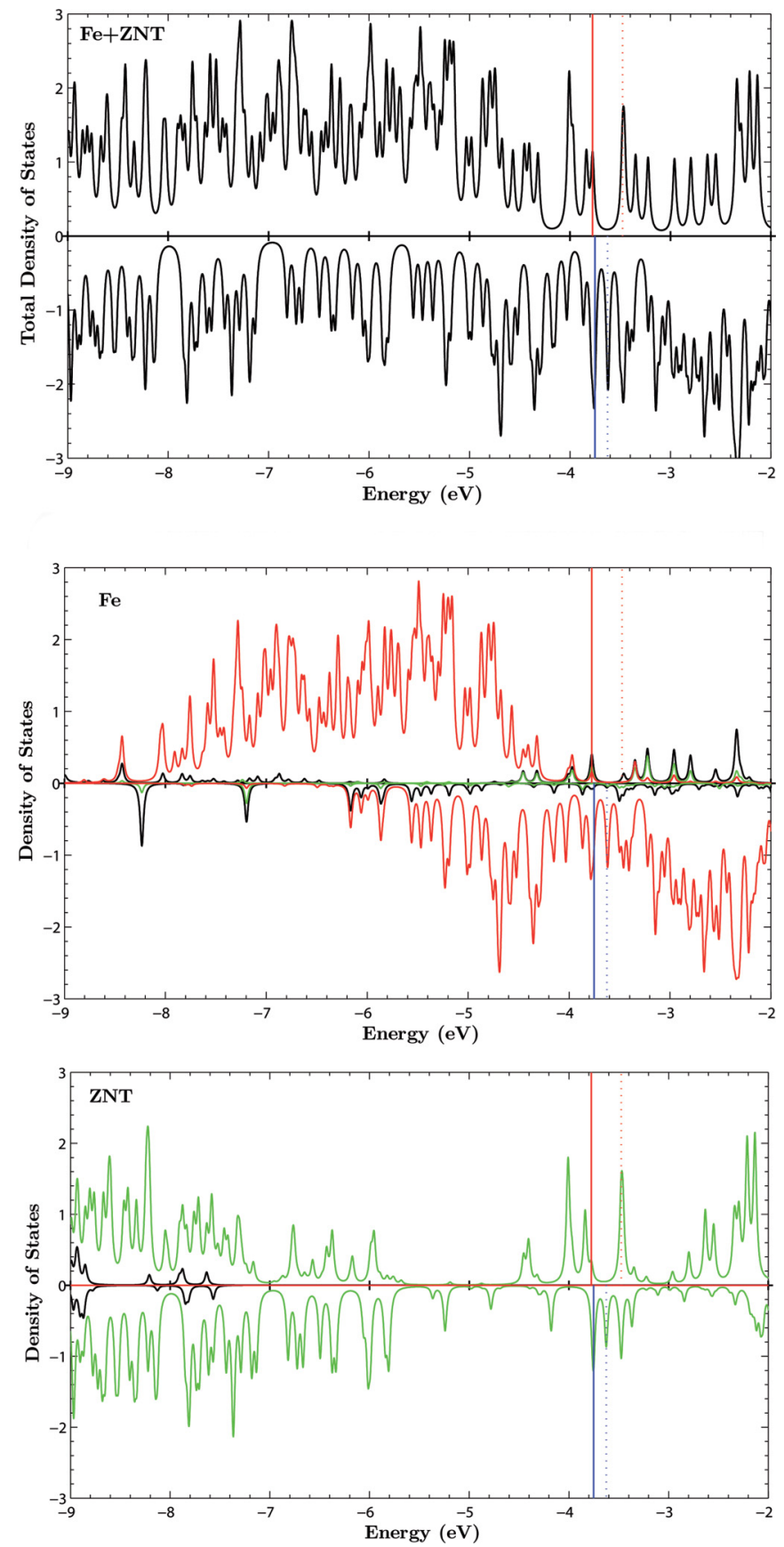

FIG. 7. (Color online) Electronic density of states, in arbitrary units, for $\mathrm{Fe}_{12} @(11,0)$ (short ZNT) in its ground state, with total magnetic moment $\mu_{T}=36 \mu_{B}$. Upper panel: total DOS. Middle and lower panels: projections of the DOS on the Fe cluster and the nanotube, respectively. In each panel, upper curves correspond to majority spin states, and lower curves to minority spin. In the middle and lower panels, red curves stand for $d$ character of the states, green curves for $p$, and black curves for $s$ character. Vertical continuous lines indicate the position of the HOMO, and dashed lines the position of the LUMO.

from the larger deformation (elongation) experienced by the iron cluster due to the smaller diameter of the $(10,0)$ nanotubes. The total magnetic moment of the system is $\mu_{T}=36 \mu_{B}$, the same as for encapsulation in the $(11,0)$ tubes, so we find again a reduction of $2 \mu_{B}$ with respect to the free iron cluster. There are electronic gaps for both spins, and the $\alpha$ and $\beta$ gaps for the short ZNT are similar to those in $\mathrm{Fe}_{12} @(11,0)$. For the longer $(10,0) \mathrm{ZNT}$, the electronic gaps indicate a trend to half-metallicity.

The total electronic DOS of $\mathrm{Fe}_{12} @(10,0)$ (long ZNT) and those projected on the Fe cluster and the nanotube are presented in Fig. 10. The general structure resembles that of $\mathrm{Fe}_{12} @(11,0)$, but there are some differences. There are no $s p$ contributions from the iron atoms to the $\alpha$-HOMO and $\alpha$-HOMO- 1 states. Such contributions were present both in pure $\mathrm{Fe}_{12}$ and in $\mathrm{Fe}_{12} @(11,0)$. The total population of the $s$ states of the encapsulated iron cluster is reduced in 1.64 electrons with respect to the free cluster. In contrast, the population of the $d$ states of the iron cluster increases in 0.54 electrons. This last change is comprised of a reduction of 0.85 electrons in the population of the $d$ - $\alpha$ states and an increase of 1.39 electrons in the population of the $d-\beta$ states. The effect of the depletion of the $s$ states of $\mathrm{Fe}$ is larger in $\mathrm{Fe}_{12} @(10,0)$ (see Fig. 10) than in $\mathrm{Fe}_{12} @(11,0)$ (see Fig. 7) due to the more intense $\mathrm{Fe}-\mathrm{C}$ interaction in the narrower $(10,0) \mathrm{ZNT}$. These results are in agreement with the trends in charge transfer from $s$ to $d$ iron orbitals obtained in previous calculations. $^{29,30}$ The detailed structure of the states near the Fermi level is shown in Fig. 11 . The $\alpha$-HOMO exhibits mainly $p$ character and is mostly localized on the ZNT. This state and the $\alpha$ state just under the HOMO are localized on the right-hand side of the ZNT. These states correspond to the occupation of the $\alpha$-LUMO orbitals of the pure ZNT shown in Fig. 4 . The $\alpha$-LUMO of $\mathrm{Fe}_{12} @(10,0)$ is a hybridized state. Sizable $p$ - $d$ hybridization is also present in both frontier orbitals of the $\beta$-spin band. The electronic DOS of $\mathrm{Fe}_{12} @(10,0)$ (short ZNT) shows the same general properties as those just described for the long ZNT.

The DOS projected on the ZNT is strongly modified with respect to the isolated tube. The same situation occurred for the $(11,0)$ tubes. To clarify the origin of these changes, we have calculated the electronic structure of the isolated long $(10,0)$ ZNT in the geometry of the tube encapsulating the $\mathrm{Fe}_{12}$ cluster. The resulting DOS is nearly identical to that of the tube in its lowest-energy configuration given in Fig. 4. Consequently, the interaction with the encapsulated cluster is responsible of the strong modification of the DOS of the nanotubes, and not the slight structural distortion of the tube. A noticeable feature of the DOS projected on the ZNT (see Figs. 7 and 10) is the emergence of spin polarization. This spinunbalanced electronic DOS of the carbon structure has been found experimentally ${ }^{19}$ by spin-polarized scanning tunneling microscopy in an organic-ferromagnetic interface.

Table III gives the changes in the magnetic moments of the cluster and of the ZNT system. The substantial changes reveal the internal redistribution of the charge between majority and minority spin states, both in the cluster and in the nanotube. For the encapsulation of the elongated $\mathrm{Fe}_{12}$ structure, the largest changes in the magnetic moments occur for $\mathrm{Fe}_{12} @(10,0)$ (long ZNT), for which the iron cluster loses $4.80 \mu_{B}$ and the carbon ZNT gains $2.80 \mu_{B}$, which results in a net reduction of $2 \mu_{B}$ in the total magnetic moment; in this case, there is a transfer of only 0.6 electrons from the cluster to the ZNT. The rearrangement of the $\alpha$ and $\beta$ charges can be seen by comparing the spin density presented in Fig. 9 with the corresponding spin 
TABLE III. Changes in the magnetic moments of the iron cluster $\Delta \mu\left(\mathrm{Fe}_{n}\right)$ and carbon nanotube $\Delta \mu(\mathrm{ZNT})$, with respect to the corresponding separated systems. For the elongated $\mathrm{Fe}_{12} @(m, 0)$ the first and second lines correspond to the short and long nanotubes, respectively.

\begin{tabular}{lcc}
\hline \hline $\mathrm{Fe}_{n} @(m, 0)$ & $\Delta \mu\left(\mathrm{Fe}_{n}\right)\left(\mu_{B}\right)$ & $\Delta \mu(\mathrm{ZNT})\left(\mu_{B}\right)$ \\
\hline $\mathrm{Fe}_{12} @(11,0)$ elongated & -3.01 & 1.01 \\
& -3.08 & 1.08 \\
$\mathrm{Fe}_{12} @(10,0)$ elongated & -3.92 & 1.92 \\
& -4.80 & 2.80 \\
$\mathrm{Fe}_{12} @(10,0)$ icosahedron & -7.38 & 1.38 \\
$\mathrm{Fe}_{12} @(10,0)$ tetrahedral chain & -4.30 & 0.30 \\
$\mathrm{Fe}_{8} @(10,0)$ two tetrahedra & -0.87 & 2.87 \\
$\mathrm{Fe}_{4} @(10,0)$ tetrahedron on edge & -0.19 & 0.19 \\
$\mathrm{Fe}_{2} @(10,0)$ & 1.12 & -1.12 \\
$\mathrm{Fe}_{(10,0) \text { top (on edge C) }}$ & -0.93 & -1.07 \\
$\mathrm{Fe} @(10,0)$ hollow & -1.17 & -0.83 \\
\hline \hline
\end{tabular}

density for the free ZNT given in the inset of Fig. 4. In Fig. 9, the two ends of the nanotube are dominated by $\alpha$ electrons (red color), whereas in Fig. 4 the left side end is dominated by $\alpha$ electrons (in red) and the right side end by $\beta$ electrons (in blue). The atomic magnetic moments are presented in Fig. 12, which shows that the atomic magnetic moments induced in the ZNT are accumulated at the two ends of the nanotube, and that the original AFM coupling of the clean ZNT is completely disturbed by the presence of the encapsulated cluster. The Fe atoms showing the largest reduction in their magnetic moments are those closest to $\mathrm{C}$ atoms.

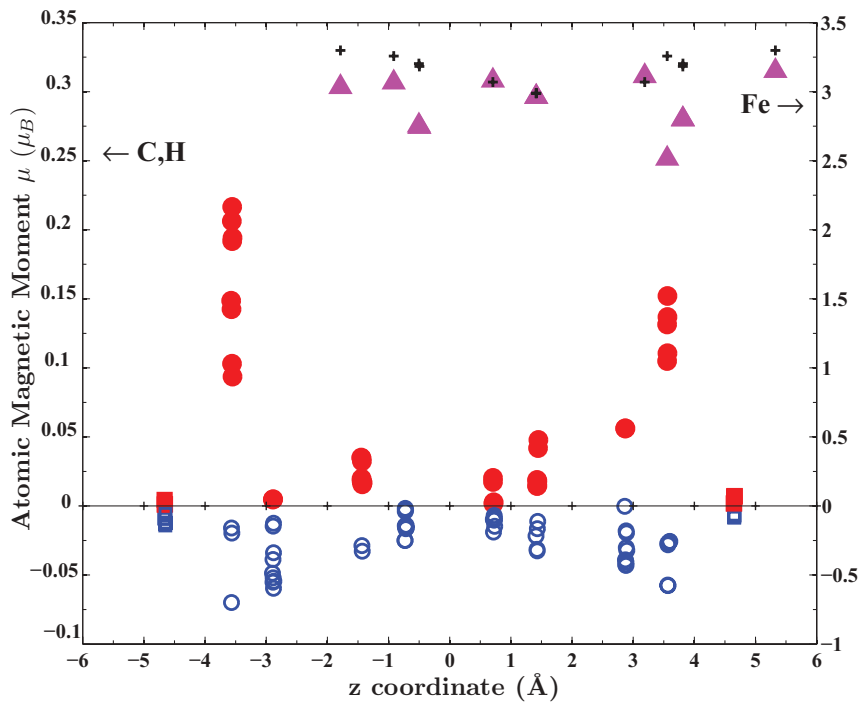

FIG. 8. (Color online) Atomic magnetic moments $\mu$ for $\mathrm{Fe}_{12} @(11,0)$ (short ZNT) as a function of the atom coordinate along the $z$ axis of the nanotube. The atomic magnetic moments of the $\mathrm{C}$ $(\mathrm{H})$ atoms with the same $z$ coordinate vary with the position of the $\mathrm{C}$ $(\mathrm{H})$ atoms on the ring. Triangles represent $\mathrm{Fe}$ atoms, circles represent $\mathrm{C}$ atoms (red circles: $\mathrm{C}$ atoms with dominant $\alpha$ spin character; blue circles: $\mathrm{C}$ atoms with dominant $\beta$ spin character), and squares on the two ends represent $\mathrm{H}$ atoms. For comparison, the atomic magnetic moments of the free $\mathrm{Fe}_{12}$ cluster (crosses) are also shown. Note the different vertical scales for Fe (right) and for $\mathrm{C}$ and $\mathrm{H}$ (left).

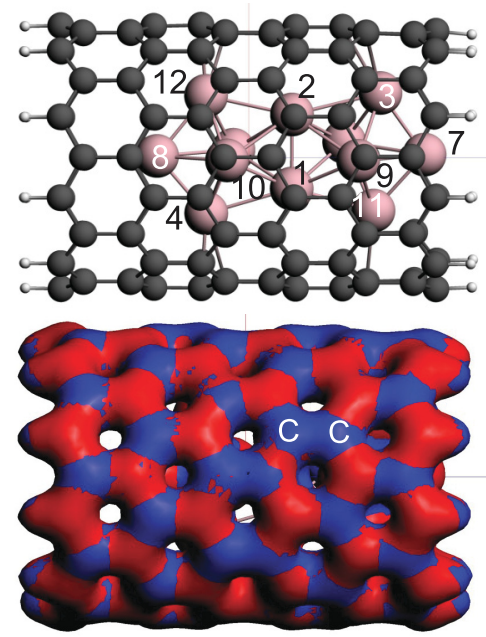

FIG. 9. (Color online) Lateral view of the equilibrium structure of $\mathrm{Fe}_{12} @(10,0)$ (long ZNT) and of the corresponding spin density. For the spin density, see caption of Fig. 3. Carbon atoms indicated by $C$ are two neighbors showing frustration of the AFM ordering.

We have also investigated the properties of the lowestenergy icosahedral structure of $\mathrm{Fe}_{12}$ encapsulated in the short $(10,0)$ tube. The results are compiled in Tables II and III. Upon full relaxation of the combined system, the structure of the iron cluster does not change significantly with respect to the free cluster and the nanotube does not experience any deformation. The approximate fivefold-symmetry axis of the iron cluster aligns with the symmetry axis of the tube. The preferred location of the iron cluster inside the tube is close to one end with the $\mathrm{Fe}$ atom of the axial vertex pointing towards the outside of the tube. The five $\mathrm{Fe}$ atoms directly linked to that vertex sit in the plane formed by the $10 \mathrm{C}$ atoms of the edge. The structure is depicted in the Supplemental Material. ${ }^{42}$ The binding energy of the encapsulated cluster, $3.172 \mathrm{eV} /$ atom, is only marginally smaller than the binding energy of the free cluster, $3.173 \mathrm{eV} /$ atom, and is larger than the binding energy of the encapsulated isomer of elongated shape $(3.052 \mathrm{eV} /$ atom). The optimal position of the cluster optimizes the interactions of the $\mathrm{Fe}$ atoms with the $\mathrm{C}$ atoms of the edge of the nanotube. The total magnetic moment is $\mu_{T}=32 \mu_{B}$, so there is a reduction in $6 \mu_{B}$ with respect to the free iron cluster. This reduction is more substantial than that experienced by the elongated isomer of $\mathrm{Fe}_{12}$ due to a more intense $\mathrm{C}-\mathrm{Fe}$ interaction in the former case. There are $10 \mathrm{Fe}$ atoms of the icosahedral isomer which have $\mathrm{C}$ neighbors at distances between 2.113 and $2.252 \AA$ and there are $40 \mathrm{C}$ atoms at those distances. These numbers are to be compared with $7 \mathrm{Fe}$ atoms and $27 \mathrm{C}$ atoms, respectively, in the case of the elongated isomer. Details on the electronic DOS of the icosahedral $\mathrm{Fe}_{12}$ encapsulated in the short $(10,0)$ tube are given in the Supplemental Material. ${ }^{42}$ The frontier orbitals exhibit an important $s p-d$ hybridization, and there is a substantial internal electronic redistribution between $\alpha$ and $\beta$ states, both in the iron cluster and in the ZNT. Similarly to the elongated isomer, the DOS projected on the ZNT is strongly modified with respect to the isolated tube. The most salient feature is the spin unbalance along the tube, which leads to a gain of magnetic moment in the tube of $1.38 \mu_{B}$. 

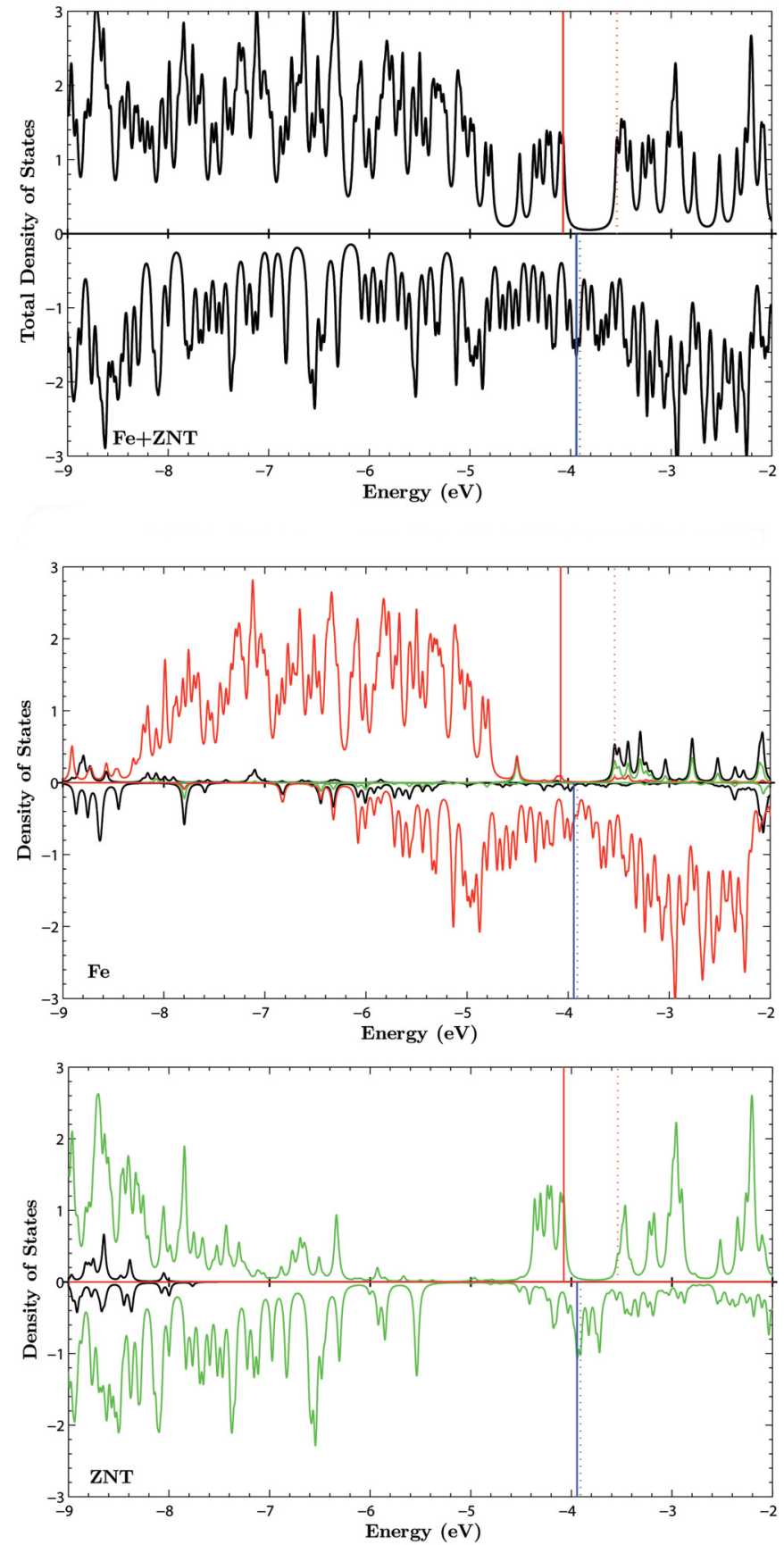

FIG. 10. (Color online) Electronic density of states, in arbitrary units, for the ground state of $\mathrm{Fe}_{12} @(10,0)$ (long ZNT) with $\mu_{T}=$ $36 \mu_{B}$. Upper panel: total DOS. The middle and lower panels show the projections of the DOS on the Fe cluster and the nanotube, respectively. In each panel, upper curves correspond to majority spin states, and lower curves to minority spin states. In the middle and lower panels, red curves stand for $d$ character of the states, green curves for $p$, and black curves for $s$ character. Vertical continuous lines indicate the position of the HOMO, and dashed lines the position of the LUMO.

\section{Small $\mathrm{Fe}_{n}(n=2,4,8)$ clusters inside the $(10,0)$ nanotube}

To shed some light on the growth process of iron clusters inside carbon nanotubes, we have studied the encapsulation of atomic iron and small $\mathrm{Fe}_{n}$ clusters $(n=2,4,8,12)$ on

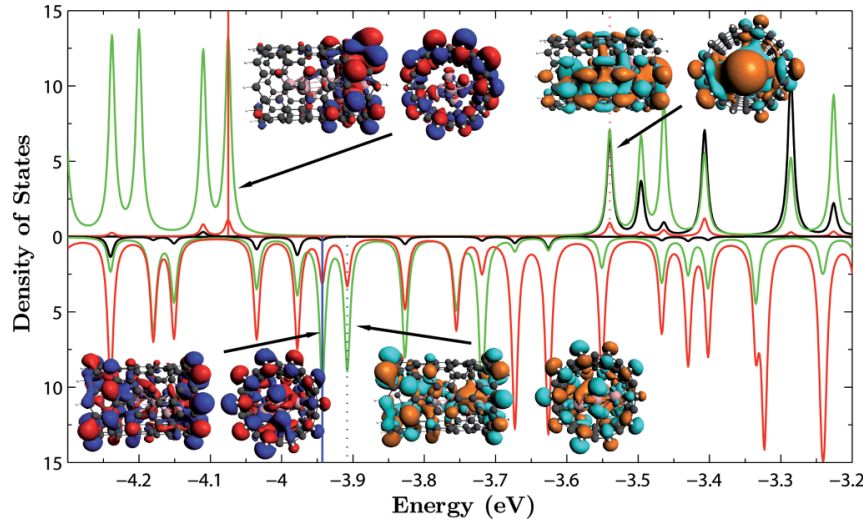

FIG. 11. (Color online) Expanded view of the total electronic density of states around the Fermi level for $\mathrm{Fe}_{12} @(10,0)$ (long ZNT), separated in $s$-like (black curves), $p$-like (green curves), and $d$-like (red curves) contributions. The Lorenztian width is $\Gamma=0.01 \mathrm{eV}$. Lateral and top views of the HOMO and LUMO orbitals are given in the insets.

the short $(10,0)$ ZNT. First, an Fe atom has been located in different positions inside the ZNT: on top of a $\mathrm{C}$ atom, in a bridge position between two $\mathrm{C}$ atoms, and in a hollow position on the center of an hexagon of $\mathrm{C}$ atoms. The highest binding energy, $1.454 \mathrm{eV}$, is obtained for the $\mathrm{Fe}$ atom on top a $\mathrm{C}$ atom of the tube edge. This reflects that the tube edges are reactive. Apart from the edges, the next highest binding energy, $0.947 \mathrm{eV}$, is obtained for the $\mathrm{Fe}$ atom in a hollow location, as is the case for an Fe atom interacting with graphene. ${ }^{29,30}$ Clearly, the encapsulation of one iron atom in a carbon nanotube is energetically favorable. There is a reduction of the total magnetic moment from $\mu_{T}=4 \mu_{B}$ in

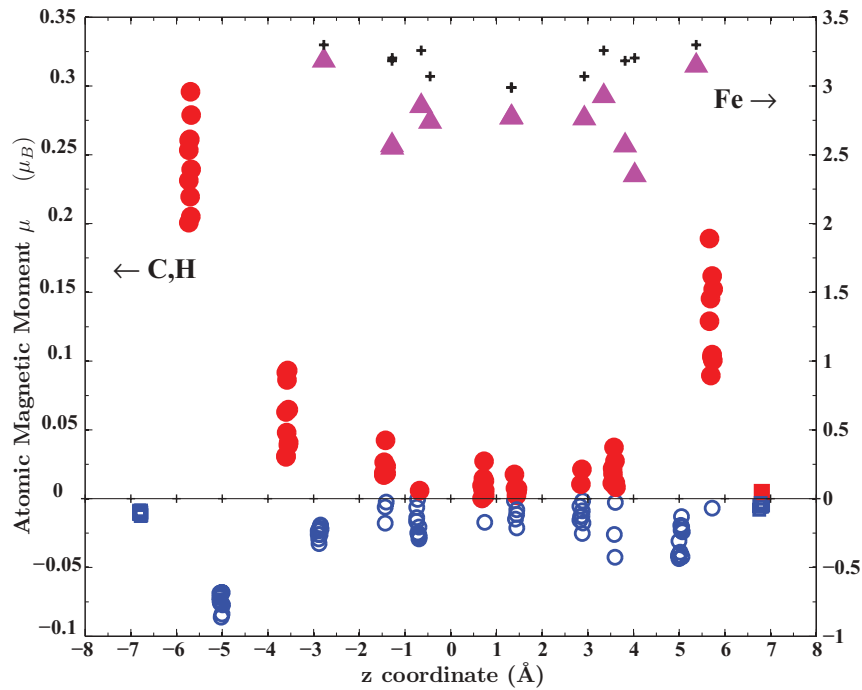

FIG. 12. (Color online) Atomic magnetic moments $\mu$ for $\mathrm{Fe}_{12} @(10,0)($ long ZNT) as a function of the atom coordinate along the $z$ axis of the nanotube. Triangles represent $\mathrm{Fe}$ atoms, circles represent $\mathrm{C}$ atoms (red circles: $\mathrm{C}$ atoms with dominant $\alpha$ character; blue circles: $\mathrm{C}$ atoms with dominant $\beta$ character), and squares on the two ends represent $\mathrm{H}$ atoms. The atomic magnetic moments of the free $\mathrm{Fe}_{12}$ cluster (crosses) are shown for comparison. Note the different vertical scales for $\mathrm{Fe}$ (right) and for $\mathrm{C}$ and $\mathrm{H}$ (left). 
the free atom to $\mu_{T}=2 \mu_{B}$ in Fe@ $(10,0)$. When a second Fe atom is added inside the tube, an $\mathrm{Fe}_{2}$ dimer is formed with an equilibrium distance $d\left(\mathrm{Fe}_{2}\right)=2.24 \AA$, somewhat larger than the equilibrium bond length, $2.02 \AA$, of the free dimer. One $\mathrm{Fe}$ atom of the dimer is bonded to a $\mathrm{C}$ atom of the edge and the other Fe atom is bonded to an internal $\mathrm{C}$ atom. The binding energy of the encapsulated $\mathrm{Fe}_{2}$ calculated from Eq. (3), $E_{b}=3.570 \mathrm{eV}$, is larger than the binding energy of the free dimer, $E_{b}^{0}\left(\mathrm{Fe}_{2}\right)=3.224 \mathrm{eV}$, so it is more favorable to form $\mathrm{Fe}_{2}$ inside the tube than as a free dimer. The dimer does not change its total magnetic moment, $\mu_{T}=6 \mu_{B}$, upon encapsulation.

The free linear $\mathrm{Fe}_{4}$ chain is a stable isomer, with all the vibrational frequencies positive, having length $6.92 \AA$, magnetic moment $\mu_{T}=14 \mu_{B}$, and binding energy $E_{b}^{0}\left(\mathrm{Fe}_{4}\right)=$ $7.154 \mathrm{eV}$, as is given in Table I. This linear chain was placed inside the short $(10,0) \mathrm{ZNT}$, parallel to the tube axis but displaced from the axis. After relaxation, the geometry of $\mathrm{Fe}_{4}$ resulted in a zigzag chain. The total magnetic moment does not change with respect to that of the free linear chain, and the binding energy is $E_{b}\left[\mathrm{Fe}_{4} @(10,0)\right]=7.532 \mathrm{eV}$. Therefore, it is favorable to encapsulate the $\mathrm{Fe}_{4}$ chain inside the nanotube, with an energy gain of $0.38 \mathrm{eV}$. One should notice, however, that the most stable structure of the free $\mathrm{Fe}_{4}$ cluster is not a linear chain, but a deformed tetrahedron (butterfly shaped) ${ }^{39}$ with $\mu_{T}=14 \mu_{B}$ and $E_{b}^{0}\left(\mathrm{Fe}_{4}\right)=9.225 \mathrm{eV}$. For this reason, we have considered an encapsulated $\mathrm{Fe}_{4}$ cluster with the deformed tetrahedral structure, placed at equal distance from the two edges of the nanotube. Relaxation modifies little the position and structure of the encapsulated cluster, the properties of which are given in Table II. The value of the magnetic moment is again $\mu_{T}=14 \mu_{B}$, and the binding energy is $E_{b}=9.352 \mathrm{eV}$. That is, encapsulated $\mathrm{Fe}_{4}$ is more stable than isolated $\mathrm{Fe}_{4}$, a result that parallels that for $\mathrm{Fe}_{2}$. Displacing the encapsulated $\mathrm{Fe}_{4}$ cluster towards one edge of the nanotube increases its binding energy because of the interaction with the carbon atoms of the edge. Three of the Fe atoms forming a face of the deformed tetrahedron are practically in the plane formed by the ten external carbon atoms of the tube, and the fourth $\mathrm{Fe}$ atom is inside the tube. In this case, $E_{b}=10.464 \mathrm{eV}$ and $\mu_{T}=14 \mu_{B}$.

As the next step in filling the $(10,0)$ nanotube, we have considered two deformed tetrahedra encapsulated inside the tube, in a relative position such that the closer edges of the two tetrahedra are orthogonal to each other. This structure for encapsulated $\mathrm{Fe}_{8}$ was also obtained by starting with two encapsulated parallel zigzag chains and relaxing this initial structure. The axis of the $\mathrm{Fe}_{8}$ cluster is parallel to the nanotube axis, but a little displaced towards the wall. The interaction between the two tetrahedra is substantial. In fact, Table II shows that the binding energy per atom of $\mathrm{Fe}_{8} @(10,0)$, $\overline{E_{b}}=2.885 \mathrm{eV}$, is higher than the binding energy per atom for two encapsulated noninteracting $\mathrm{Fe}_{4}$ clusters, 2.338 or $2.616 \mathrm{eV}$.

We have also explored an encapsulated chain formed by three $\mathrm{Fe}_{4}$ tetrahedral units. The three tetrahedra are placed with the same orientation and aligned along a $\mathrm{C}_{2}$ axis of the tetrahedra. The interaction between the tetrahedra is substantial and there is an energy gain of $4.25 \mathrm{eV}$ when the encapsulated tetrahedral chain is formed from tetrahedral $\mathrm{Fe}_{8}$ and $\mathrm{Fe}_{4}$ (centered) encapsulated clusters. The binding energy per iron atom of the encapsulated tetrahedral chain (3.057 eV/atom) is only slightly lower than that of the lowest-energy encapsulated icosahedron. This shows that the tetrahedral type of growth is competitive with respect to other structures. The magnetic moment of the tetrahedral chain $\mathrm{Fe}_{12} @(10,0), \mu_{T}=34 \mu_{B}$, is only reduced by $4 \mu_{B}$ with respect to the moment of the free iron cluster. The total moment is intermediate between those of the encapsulated elongated isomer and the encapsulated icosahedral structure. Our results reveal a tendency of the encapsulated atoms and small clusters to aggregate, forming elongated clusters and wires in the geometrical confinement imposed by the nanotube. Moreover, the nanotube preserves, to a large extent, the magnetic moment of the encapsulated iron cluster. Details on the actual geometry, the electronic DOS, and of the magnetic structure of the tetrahedral chain $\mathrm{Fe}_{12}$ encapsulated in the short $(10,0)$ tube are given in the Supplemental Material. ${ }^{42}$

The properties of free iron clusters are summarized in Table I, and those for encapsulated clusters in Tables II and III. Encapsulation of atoms, dimers, and tetramers is energetically favorable in the $(10,0)$ nanotube. Once inside, those atoms and clusters tend to aggregate, and formation of wires based on the $\mathrm{Fe}_{4}$ tetrahedron is plausible for long nanotubes, as assumed previously. ${ }^{21}$ The binding energies per atom of the encapsulated iron clusters increase as a function of cluster size, which reveals the tendency of the iron atoms and small clusters to aggregate into larger elongated clusters inside the tubes. This justifies the iron-filled tubes produced experimentally. $6,7,40$

Free iron clusters exhibit large magnetic moments (see Table I). The magnetic moments of the encapsulated iron clusters, shown in Table III, are reduced with respect to the moments of the free clusters, except for $\mathrm{Fe}_{2}$, whereas there is an enhancement in the net magnetization of the ZNT.

\section{SUMMARY AND CONCLUSIONS}

We have performed DFT calculations of the electronic structure of different isomers of the $\mathrm{Fe}_{12}$ cluster located inside finite pieces of the $(11,0)$ and the $(10,0)$ nanotubes of two different lengths. Moreover, we have also studied the encapsulation of $\mathrm{Fe}$ atoms and small $\mathrm{Fe}_{n}$ clusters $(n=2,4,8)$ in a short $(10,0)$ tube. Motivated by the constraints imposed by the cylindrical geometry of a narrow nanotube, we have first considered the encapsulation of an isomer of $\mathrm{Fe}_{12}$ with an elongated structure. We have also considered the encapsulation of the lowest-energy, icosahedral structure of $\mathrm{Fe}_{12}$. The free elongated $\mathrm{Fe}_{12}$ isomer presents a strong ferromagnetic spin coupling giving rise to a large magnetic moment $\mu_{T}=38 \mu_{B}$. The well-known properties of the electronic structure of the isolated ZNTs, such as the antiferromagnetic arrangement of the spin distribution along the direction of the tube axis, with $\mu_{T}=0 \mu_{B}$, the spatial localization of states with energies around the Fermi level, the spin compensation of the electronic DOS at any energy, and the electronic gaps, are substantially modified by the interaction with the encapsulated iron aggregates. Comparatively, the distortion in the DOS of the iron cluster is much smaller, preserving the strong ferromagnetic character. There is some $p$ - $d$ hybridization between the nanotube and the cluster states, mainly around 
the Fermi level. The LUMO of the pristine ZNT becomes partially occupied due to charge transfer from the cluster to the nanotube.

The magnetic moment of the encapsulated $\mathrm{Fe}_{12}$ cluster is reduced with respect to the free cluster by an amount between 3.0 and $7.4 \mu_{B}$, depending on the diameter and length of the nanotube. This reduction is driven partially by a small charge transfer from the iron cluster to the ZNT, and mostly by the internal redistribution of the spin-up and spin-down electronic charges of the cluster. In contrast, there is an increase in the magnetic moment of the ZNTs, ranging from 1.0 to $2.8 \mu_{B}$. The changes in the magnetic moments of the iron cluster and tube do not compensate each other and the net effect is a small reduction in the range from 2 to $6 \mu_{B}$ in the total magnetic moment.

Encapsulation of atomic $\mathrm{Fe}, \mathrm{Fe}_{2}$, and $\mathrm{Fe}_{4}$ inside carbon nanotubes is energetically favorable. Positions near the nanotube edges are preferred compared to positions deeper inside the tube because the edges are very active. The same conclusion has been reached for the larger $\mathrm{Fe}_{12}$ cluster, which exhibits a slightly higher binding energy in the shorter than in the longer ZNTs due to the direct interaction with the two edges. Iron atoms and small iron clusters inside carbon nanotubes exhibit a strong tendency to aggregate. Because of the constraints imposed by the cylindrical structure of narrow nanotubes, the encapsulated clusters exhibit elongated structures. However, the structure of lowest-energy results from the encapsulation of $\mathrm{Fe}_{12}$ preserving its ground-state icosahedral structure. For the nanotube diameters explored in this work, the growth of a nanowire formed by distorted $\mathrm{Fe}_{4}$ tetrahedral units properly oriented looks promising.

To further understand the magnetic properties of the interface between a carbon material and a ferromagnetic material, and to clarify the effects due to $p$ - $d$ hybridization, it will be interesting to study transition-metal clusters deposited on synthetic nanographenes with zigzag boundaries, ${ }^{43}$ which in their triangular and hexagonal forms have been predicted to have permanent magnetic moments. ${ }^{44}$

In general, free metallic magnetic nanoparticles are oxide coated, but the encapsulation of Fe clusters and nanowires in carbon nanotubes protects these metallic nanoparticles from the oxidating environment. A result of our study is that the interaction of the encapsulated iron cluster with the carbon nanotube preserves, to a large extent, the strong ferromagnetic coupling of the iron cluster, with only a minor reduction of the magnetic moment. Therefore, iron clusters encapsulated in carbon nanotubes may be promising in different applications.

\section{ACKNOWLEDGMENTS}

Work supported by MICINN of Spain and the European Regional Development Fund (Projects No. MAT2008-06483C03-01, No. MAT2008-06483-C03-03, and No. MAT201122781), and by Junta de Castilla y León (Project No. VA158A11-2). *angel.mananes@unican.es

${ }^{1}$ A. Taylor, K. Lipert, K. Krämer, S. Hampel, S. Füssel, A. Meye,

R. Klingeler, M. Ritschel, A. Leonhardt, B. Büchner et al., J. Nanosci. Nanotechnol. 9, 5709 (2009).

${ }^{2}$ S. A. Wolf, D. D. Awschalom, R. A. Buhrman, J. M. Daughton, S. von Molnár, M. L. Roukes, A. Y. Chtchelkanova, and D. M. Treger, Science 294, 1488 (2001).

${ }^{3}$ X. Blase, C. Adessi, B. Biel, A. López-Bezanilla, M.-V. FernándezSerra, E. R. Margine, F. Triozon, and S. Roche, Phys. Status Solidi B 247, 2962 (2010)

${ }^{4}$ F. Wolny, T. Mühl, U. Weissker, K. Lipert, J. Schumann, A. Leonhardt, and B. Büchner, Nanotechnology 21, 435501 (2010).

${ }^{5}$ A. Winkler, T. Mühl, S. Menzel, R. Kozhuharova-Koseva, S. Hampel, A. Leonhardt, and B. Büchner, J. Appl. Phys. 99, 104905 (2006).

${ }^{6}$ U. K. Gautam, P. M. F. J. Costa, Y. Bando, X. Fang, L. Li, M. Imura, and D. Golberg, Sci. Technol. Adv. Mater. 11, 54501 (2010).

${ }^{7}$ A. A. Eliseev, M. V. Kharlamova, M. V. Chernysheva, A. V. Lukashin, Y. D. Tretyakov, A. S. Kumskov, and N. A. Kiselev, Russ. Chem. Rev. 78, 833 (2009).

${ }^{8}$ D. Kondo, K. Yagi, M. Sato, Y. Awano, S. Sato, and N. Yokoyama, Chem. Phys. Lett. 514, 294 (2011).

${ }^{9}$ D. L. Peng, X. Zhao, S. Inoue, Y. Ando, and K. Sumiyama, J. Magn. Magn. Mater. 292, 143 (2005).

${ }^{10}$ N. Grobert, W. K. Hsu, Y. Q. Zhu, J. Hare, H. W. Kroto, D. R. M. Walton, M. Terrones, H. Terrones, P. Redlich, M. Rühle et al., Appl. Phys. Lett. 75, 3363 (1999).
${ }^{11}$ J. Kohanoff, Electronic Structure Calculations for Solids and Molecules: Theory and Computational Methods (Cambridge University Press, Cambridge, UK, 2006).

${ }^{12}$ A. J. Du, Z. H. Zhu, C. H. Sun, Y. Chen, G. Q. Lu, and S. C. Smith, Chem. Phys. Lett. 468, 257 (2009).

${ }^{13}$ A. J. Du, Y. Chen, G. Q. Lu, and S. C. Smith, Appl. Phys. Lett. 93, 73101 (2008).

${ }^{14}$ O. Hod and G. Scuseria, ACS Nano 2, 2243 (2008).

${ }^{15}$ A. Mañanes, F. Duque, A. Ayuela, M. J. López, and J. A. Alonso, Phys. Rev. B 78, 035432 (2008).

${ }^{16}$ Z. Chen, D. Jiang, X. Lu, H. F. Bettinger, S. Dai, P. von Ragué Schleyer, and K. N. Houk, Org. Lett. 9, 5449 (2007).

${ }^{17}$ O. Hod, V. Barone, and G. Scuseria, Phys. Rev. B 77, 035411 (2008).

${ }^{18}$ F. Muñoz-Rojas, J. Fernández-Rossier, and J. J. Palacios, Phys. Rev. Lett. 102, 136810 (2009).

${ }^{19}$ N. Atodiresei, J. Brede, P. Lazić, V. Caciuc, G. Hoffmann, R. Wiesendanger, and S. Blügel, Phys. Rev. Lett. 105, 066601 (2010).

${ }^{20}$ S. F. Wang, Y. Zhang, L. Y. Chen, J. M. Zhang, and K. W. Xu, Phys. Status Solidi A 208, 97 (2010).

${ }^{21}$ C. Jo and J. I. Lee, J. Magn. Magn. Mater. 320, 3256 (2008).

${ }^{22}$ V. V. Ivanovskaya, C. Köhler, and G. Seifert, Phys. Rev. B 75, 075410 (2007).

${ }^{23}$ S. Yuan, Y. Kong, F. Wen, and F. Li, J. Phys.: Condens. Matter 19, 466203 (2007). 
${ }^{24}$ M. Weissmann, G. García, M. Kiwi, R. Ramírez, and C.-C. Fu, Phys. Rev. B 73, 125435 (2006).

${ }^{25}$ Y.-J. Kang, J. Choi, C.-Y. Moon, and K. J. Chang, Phys. Rev. B 71, 115441 (2005).

${ }^{26}$ Y. Yagi, T. M. Briere, M. H. F. Sluiter, V. Kumar, A. A. Farajian, and Y. Kawazoe, Phys. Rev. B 69, 075414 (2004).

${ }^{27}$ S. B. Fagan, R. Mota, A. J. R. da Silva, and A. Fazzio, Phys. Rev. B 67, 205414 (2003).

${ }^{28}$ C. K. Yang, J. Zhao, and J. P. Lu, Phys. Rev. Lett. 90, 257203 (2003).

${ }^{29}$ C. Cao, M. Wu, J. Jiang, and H.-P. Cheng, Phys. Rev. B 81, 205424 (2010).

${ }^{30}$ K. T. Chan, J. B. Neaton, and M. L. Cohen, Phys. Rev. B 77, 235430 (2008).

${ }^{31}$ X. Zou, G. Zhou, J. Li, B. Yan, J. Wu, B.-L. Gu, and W. Duan, Appl. Phys. Lett. 94, 193106 (2009).

${ }^{32}$ N. Fujima and T. Oda, Phys. Rev. B 71, 115412 (2005).

${ }^{33}$ E. Durgun and S. Ciraci, Phys. Rev. B 74, 125404 (2006).

${ }^{34}$ G. te Velde, F. M. Bickelhaupt, E. J. Baerends, C. Fonseca-Guerra, S. J. A. van Gisbergen, J. G. Snijders, and T. Ziegler, J. Comput. Chem. 22, 931 (2001).
${ }^{35}$ C. Fonseca-Guerra, J. G. Snijders, G. te Velde, and E. J. Baerends, Theor. Chem. Acc. 99, 391 (1998).

${ }^{36}$ Y. Zhang and W. Yang, Phys. Rev. Lett. 80, 890 (1998).

${ }^{37}$ J. Bansmann, S. H. Baker, C. Binns, J. A. Blackman, J. P. Bucher, J. Dorantes-Dávila, V. Dupuis, L. Favre, D. Kechrakos, A. Kleibert et al., Surf. Sci. Rep. 56, 189 (2005).

${ }^{38}$ J. A. Alonso, Chem. Rev. 100, 637 (2000).

${ }^{39}$ O. Diéguez, M. M. G. Alemany, C. Rey, P. Ordejón, and L. J. Gallego, Phys. Rev. B 63, 205407 (2001).

${ }^{40}$ N. Y. Jin-Phillipp and M. Rühle, Phys. Rev. B 70, 245421 (2004).

${ }^{41}$ J. A. Alonso and N. H. March, Electrons in Metals and Alloys (Academic, London, 1989).

${ }^{42}$ See Supplemental Material at http://link.aps.org/supplemental/ 10.1103/PhysRevB.87.085402 for the figures corresponding to the structure, spin density, electronic DOS, HOMO and LUMO orbitals, and atomic magnetic moments of $\mathrm{Fe}_{12}$, icosahedral, and tetrahedral chains, encapsulated in the short $(10,0) \mathrm{ZNT}$.

${ }^{43}$ K. A. Ritter and J. W. Lyding, Nat. Mater. 8, 235 (2009).

${ }^{44}$ J. Fernández-Rossier and J. J. Palacios, Phys. Rev. Lett. 99, 177204 (2007). 